\title{
Nucleon-nucleon interaction: Central potential and pion production
}

\author{
C. M. Maekawa, ${ }^{1}$ J. C. Pupin, ${ }^{3,2}$ and M. R. Robilotta ${ }^{2}$ \\ ${ }^{1}$ Kellogg Radiation Laboratory, 106-38 California Institute of Technology, Pasadena, California 91125 \\ ${ }^{2}$ Nuclear Theory and Elementary Particle Phenomenology Group, Instituto de Física, Universidade de São Paulo, \\ C.P. 66 318, 05315-970, São Paulo, SP, Brazil \\ ${ }^{3}$ Instituto de Física Teórica, Universidade Estadual Paulista, 01405-900, Rua Pamplona 145, São Paulo, SP, Brazil
}

(Received 25 October 1999; published 27 April 2000)

\begin{abstract}
We show that the tail of the chiral two-pion exchange nucleon-nucleon potential is proportional to the pion-nucleon $(\pi N)$ scalar form factor and discuss how it can be translated into effective scalar meson interactions. We then construct a kernel for the process $N N \rightarrow \pi N N$, due to the exchange of two pions, which may be used in either three-body forces or pion production in $N N$ scattering. Our final expression involves a partial cancellation among three terms, due to chiral symmetry, but the net result is still important. We also find that, at large internucleon distances, the kernel has the same spatial dependence as the central $N N$ potential and we produce expressions relating these processes directly.
\end{abstract}

PACS number(s): 13.75.Cs, 13.75.Gx, 11.30.Rd

\section{INTRODUCTION}

The description of pion production in nucleon-nucleon $(N N)$ interactions near threshold is a traditional problem in hadron physics. In recent times interest in it was renewed, due to a wealth of precise experimental data: $n p \rightarrow d \pi^{0}$ [1], and $p p \rightarrow p p \pi^{0}[2,3], p p \rightarrow d \pi^{+}[4,5]$, and $p p \rightarrow p n \pi^{+}[6]$. On the theoretical side, the availability of chiral perturbation theory (ChPT) allowed the problem to be tackled in a systematic manner. However, in spite of all the effort made, a satisfactory picture is still not available.

There are two classes of interactions involved in this process, associated with either nucleon correlations or the emission of the external pion. In the procedure developed by Koltun and Reitan [7], these interactions are encompassed in wave functions and interaction kernels. The former correspond to solutions of the Schrödinger equation with realistic potentials, whereas the latter are described by models based on Feynman diagrams.

In the discussion of the production kernel, one usually distinguishes between long and short range contributions. The former are shown in Fig. 1, where the first diagram represents the impulse approximation and the second the pion rescattering term. These two processes were considered by Koltun and Reitan [7] in their description of the $\pi^{0}$ channel, but the corresponding cross section proved to underestimate recent data [1-3] by a factor of 5 [8]. The rescattering term used in that work came from on-shell $\pi N$ amplitudes, whereas the pion exchanged in diagram 1(b) is off shell. Models which take pion virtuality into account enhance the cross section and tend to reduce the underprediction [9-12]. Heavy baryon ChPT calculations [13-15] also stressed the importance of this rescattering term at leading order. However, in these works the rescattering and impulse terms came out with opposite signs and the net result was again smaller than in phenomenological calculations [16]. Hence other mechanisms are needed to improve the description.

The next natural step concerns shorter range interactions, especially those involving two pions. The treatment of uncorrelated two-pion exchange is rather complex and, in the case of pion production, this part of the interaction has been described by effective heavy meson exchanges. Their contributions correspond to the last diagram of Fig. 1, known as $z$ graph, since positive frequency nucleon propagation, already included in the wave function, is subtracted. The inclusion of $\sigma, \omega$, and $\rho$ mesons, either explicitly [12] or in a general axial current density [17], gave rise to good fits to the $p p$ $\rightarrow p p \pi^{0}$ cross section at threshold. However, the study of relativistic effects in $\pi^{0}$ production [18] demonstrated that $z$-graph effects are small and hence heavy mesons in ChPT $[13,14]$ do not give rise to the required increase in cross sections. Extension to other channels $\left(\pi^{+}\right.$and $\left.\pi^{-}\right)$, with inclusion of nucleon resonances $[15,19,20]$, also did not improve the situation.

Some time ago, Coon, Peña, and Riska [21] produced a three-body potential based on the exchanges of a pion and a scalar meson, which proved to be able to reduce the gap between theory and experiment for the binding energy of trinuclei. Later on, we derived an equivalent result, using a nonlinear Lagrangian, which included an effective chiral scalar meson coupled to nucleons [22]. In that work, the effective field was designed to simulate the two-pion exchange potential. We stress that the exchange of two uncorrelated pions, formulated in the framework of chiral symmetry and including delta degrees of freedom, explains quite well the tail of the scalar-isoscalar nucleon-nucleon potential [23-25] and there is no need at all for a true scalar meson to describe that channel. On the other hand, the treatment of uncorrelated two-pion exchange requires the calculation of many Feynman diagrams and, in problems where one is more con-

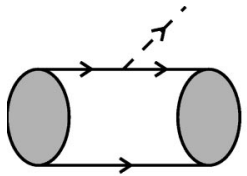

(a)

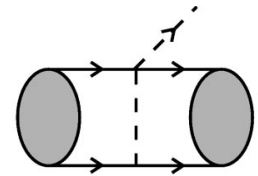

(b)

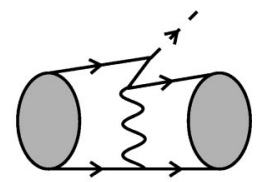

(c)
FIG. 1. Contributions to the process $N N \rightarrow \pi N N$ : (a) impulse, (b) rescattering, and (c) $z$ graphs; nucleons, pions, and heavier mesons are represented by solid, dashed, and wavy lines. 


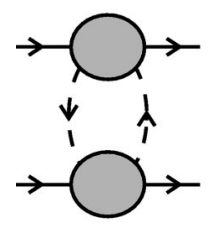

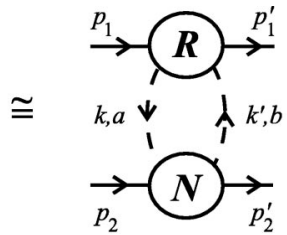

(a)

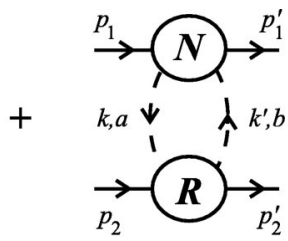

(b)
FIG. 2. Leading contributions to the process $N N \rightarrow N N$.

cerned with simplicity than with fine details, it may be useful to replace all processes associated with the scalar-isoscalar channel by a single effective field. In this conceptual framework, our Lagrangian gave rise to a strong pion-scalarnucleon contact interaction, which corresponds to the kernel for the reaction $N N \rightarrow \pi N N$ due to the exchange of two pions. This kernel was then applied to the $\pi^{0}$ and $\pi^{+}$production channels and theoretical results were found to be comparable to the experimental ones [26]. Recently, calculations based on both relativistic [27] and heavy baryon ChPT [28] dealt with such a transition operator and large contributions were again found, involving several cancellations.

The purpose of the present work is twofold. In Sec. II, we discuss the relationship between the actual tail of the twopion exchange potential and that provided by an effective scalar meson. The pion production kernel is considered in Sec. III and, in order to determine the role of chiral cancellations, we study the leading term, constructed by means of the $\pi N \rightarrow \pi N$ and $\pi N \rightarrow \pi \pi N$ subamplitudes. Our results are based on a partial cancellation, involving three large factors. We also find that, at large internucleon distances, the kernel has the same spatial dependence as the central $N N$ potential and hence, in Sec. IV, we produce expressions relating these interactions directly. Finally, in Sec. V we present a summary and conclusions.

\section{CENTRAL POTENTIAL}

The two-pion exchange potential (TPEP) is closely related to $\pi N$ scattering. The isoscalar amplitude for the process $N N \rightarrow N N$ is represented in Fig. 2 and given by

$$
\mathcal{T}^{S}=-\frac{i}{2 !} \int \frac{d^{4} Q}{(2 \pi)^{4}} \frac{3\left[T^{+}\right]^{(1)}\left[T^{+}\right]^{(2)}}{\left(k^{2}-\mu^{2}\right)\left(k^{\prime 2}-\mu^{2}\right)}
$$

where $T^{+}$is the isospin symmetric part of amplitude for the process $\pi^{a} N \rightarrow \pi^{b} N$ and $Q=\left(k^{\prime}+k\right) / 2$.

In recent times, chiral symmetry has been systematically applied to this problem and one has learned [23-25] that it is convenient to separate $T^{+}$into a contribution $T_{N}^{+}$, due only to pion-nucleon interactions, and a remainder $T_{R}^{+}$, involving other degrees of freedom. One then writes symbolically $\left[T^{+}\right]=\left[T_{N}^{+}\right]+\left[T_{R}^{+}\right]$for each nucleon and the potential is then proportional to $\left[T^{+}\right]^{(1)}\left[T^{+}\right]^{(2)}=\left[T_{N}^{+}\right]^{(1)}\left[T_{N}^{+}\right]^{(2)}$ $+\left\{\left[T_{N}^{+}\right]^{(1)}\left[T_{R}^{+}\right]^{(2)}+\left[T_{R}^{+}\right]^{(1)}\left[T_{N}^{+}\right]^{(2)}\right\}+\left[T_{R}^{+}\right]^{(1)}\left[T_{R}^{+}\right]^{(2)}$. The numerical study of these contributions has shown that the term within curly brackets is largely dominant [24] and due to the subamplitudes

$$
\begin{gathered}
T_{N}^{+}=\frac{g^{2}}{m} \bar{u}\left\{1-\left[\frac{m}{(p+k)^{2}-m^{2}}-\frac{m}{\left(p-k^{\prime}\right)^{2}-m^{2}}\right] \Phi\right\} u, \\
T_{R}^{+}=\bar{A}^{+}\left(\nu=0, t=4 \mu^{2}\right) \bar{u} u=\frac{\alpha_{00}^{+}}{\mu} \bar{u} u,
\end{gathered}
$$

where $g$ is the $\pi N$ coupling constant, $m$ is the nucleon mass, and the bar over the isospin even $\pi N$ subamplitude $A^{+}$indicates the subtraction of the pseudoscalar Born term. The constant $\alpha_{00}^{+}$may be expressed as a combination [24] of $\pi N$ subthreshold coefficients [29]. The leading contribution to $\mathcal{T}^{S}$ is written as

$$
\mathcal{T}^{S} \cong 3 \frac{\alpha_{00}^{+}}{\mu}[\bar{u} u]^{(1)}\left[\Gamma_{N}^{+}\right]^{(2)}+(1 \leftrightarrow 2),
$$

where

$$
\begin{aligned}
{\left[\Gamma_{N}^{+}\right]^{(2)}=} & -\frac{i}{2} \int \frac{d^{4} Q}{(2 \pi)^{4}} \\
& \times \frac{\left[T_{N}^{+}\right]^{(2)}}{\left[(Q-\Delta / 2)^{2}-\mu^{2}\right]\left[(Q+\Delta / 2)^{2}-\mu^{2}\right]},
\end{aligned}
$$

with $\Delta=\left(k^{\prime}-k\right)$. Using Eq. (2), we have

$$
\begin{aligned}
{\left[\Gamma_{N}^{+}\right]^{(2)=} } & -\frac{i}{2} \frac{g^{2}}{m}\left\{[\bar{u} u]^{(2)} \int \frac{d^{4} Q}{(2 \pi)^{4}} \frac{1}{\left[(Q-\Delta / 2)^{2}-\mu^{2}\right]\left[(Q+\Delta / 2)^{2}-\mu^{2}\right]}\right. \\
& \left.-\left[\bar{u} \gamma_{\mu} u\right]^{(2)} \int \frac{d^{4} Q}{(2 \pi)^{4}} \frac{2 m Q^{\mu}}{\left[(Q-\Delta / 2)^{2}-\mu^{2}\right]\left[(Q+\Delta / 2)^{2}-\mu^{2}\right]\left[Q^{2}+2 m V_{2} \cdot Q-\Delta^{2} / 4\right]}\right\} \\
= & \frac{1}{2} \frac{g^{2}}{m} \frac{1}{(4 \pi)^{2}}\left[J_{c, c}(t)-J_{c, s N}^{(1)}(t)\right][\bar{u} u]^{(2)} .
\end{aligned}
$$


In deriving this result we used $V_{2}=\left(p_{2}^{\prime}+p_{2}\right) / 2 m$ and the symmetry of the integrand under $Q \rightarrow-Q$. The functions $J$, defined in Ref. [30], are given by

$$
\begin{gathered}
J_{c, c}(t)=C(d, \Lambda)-\mu^{2} \int_{0}^{1} d \alpha \int_{0}^{1} \frac{d \beta}{\beta} \frac{\lambda^{2}}{t-\lambda^{2} \mu^{2}}, \\
J_{c, s N}^{(1)}(t)=-2 m^{2} \int_{0}^{1} d \alpha \frac{1-\alpha}{\alpha} \int_{0}^{1} d \beta \frac{1-\beta}{\beta} \frac{1}{t-\eta^{2} \mu^{2}},
\end{gathered}
$$

with $t=\Delta^{2}$ and

$$
\begin{gathered}
\lambda^{2}=1 /[\alpha(1-\alpha) \beta], \\
\eta^{2}=\left[(1-\alpha)^{2}(1-\beta)^{2} m^{2} / \mu^{2}+1\right. \\
-(1-\alpha)(1-\beta)] /[\alpha(1-\alpha) \beta] .
\end{gathered}
$$

The integral $J_{c, c}$ contains a function $C(d, \Lambda)$, where $d$ is the number of space-time dimensions and $\Lambda$ is the mass scale that arises in dimensional regularization. In the limit $d \rightarrow 4$ this function becomes divergent and needs to be removed by renormalization. We neglect this contribution because it has zero range and overlaps with other short distance effects not considered here.

The function $\left[\Gamma_{N}^{+}\right]$is related to the scalar form factor $\sigma(t)$ by $\left\langle p^{\prime}\left|\mathcal{L}_{s b}\right| p\right\rangle=-\sigma(t)[\bar{u} u]$, where $\mathcal{L}_{s b}$ is the symmetry breaking Lagrangian. Its long range structure, as discussed by Gasser, Sainio, and Svarc [31], is associated with diagrams 2(a) and 2(b), and hence, in our notation, one has the equivalence

$$
\sigma(t)[\bar{u} u]=3 \mu^{2}\left[\Gamma_{N}^{+}\right],
$$

which is valid for large distances. This allows the asymptotic scalar potential to be written as

$$
\mathcal{T}^{S} \cong 2 \frac{\alpha_{00}^{+}}{\mu} \frac{\sigma(t)}{\mu^{2}}[\bar{u} u]^{(1)}[\bar{u} u]^{(2)}
$$

This result is interesting because it sheds light into the structure of the interaction. The picture that emerges is that of a nucleon, acting as a scalar source, disturbing the pion cloud of the other. The function $\sigma(t)$ is related to the $\pi N$ $\sigma$-term by $\sigma(0)=\sigma_{N}$ and its value at the Cheng-Dashen point $t=2 \mu^{2}$ may be extracted from experiment.

In some situations, it may be useful to use an effective parametrized version of $\sigma(t)$. In this case, the $t$ dependence of Eqs. (7) and (8) suggests that one should use the form

$$
\sigma(t) \approx-\frac{c}{t-m_{s}^{2}},
$$

where the free parameters $c$ and $m_{s}$ may be written in terms of $\sigma\left(2 \mu^{2}\right)$ and $\sigma(0)$ as

$$
c=\sigma(0) m_{s}^{2},
$$

TABLE I. Predictions for $g_{s}$ and $m_{s}$ from Eqs. (14) and (15).

\begin{tabular}{lcccc}
\hline \hline$\alpha_{00}^{+}$ & $\sigma\left(2 \mu^{2}\right)(\mathrm{MeV})$ & {$\left[\sigma\left(2 \mu^{2}\right)-\sigma(0)\right](\mathrm{MeV})$} & $g_{s}$ & $m_{s}(\mathrm{MeV})$ \\
\hline $3.68^{\mathrm{a}}$ & $60^{\mathrm{c}}$ & $7.3^{\mathrm{b}}$ & 7.22 & 564 \\
$3.68^{\mathrm{a}}$ & $60^{\mathrm{c}}$ & $15^{\mathrm{c}}$ & 4.36 & 393 \\
$6.74^{\mathrm{d}}$ & $88^{\mathrm{d}}$ & $15^{\mathrm{c}}$ & 9.09 & 478 \\
$4.61^{\mathrm{e}}$ & $90^{\mathrm{e}}$ & $15^{\mathrm{c}}$ & 7.71 & 483 \\
\hline \hline
\end{tabular}

${ }^{\mathrm{a}}$ Reference [29].

${ }^{\mathrm{b}}$ Reference [31].

${ }^{\mathrm{c}}$ Reference [32].

${ }^{\mathrm{d}}$ Reference [33].

${ }^{\mathrm{e}}$ Reference [34].

$$
m_{s}^{2}=\frac{2 \sigma\left(2 \mu^{2}\right)}{\sigma\left(2 \mu^{2}\right)-\sigma(0)} \mu^{2} .
$$

The coupling constant of this effective scalar state to nucleons may be obtained by comparing Eq. (12) with

$$
\mathcal{T}^{S} \approx-\frac{g_{s}^{2}}{t-m_{s}^{2}}[\bar{u} u]^{(1)}[\bar{u} u]^{(2)}
$$

and one has

$$
g_{s}^{2} \approx 2 \alpha_{00}^{+} \frac{m_{s}^{2} \sigma(0)}{\mu^{3}} .
$$

In Table I we display the values of $g_{s}$ and $m_{s}$ obtained from input factors found in the recent literature. In most cases, the scalar mass is close to that used in the Bonn potential [35], but the coupling constant is smaller. We would like to stress, however, that the purpose of this exercise is not to predict these values. Instead, it is to show that the actual asymptotic exchange of two uncorrelated pions may be naturally simulated in terms of an effective scalar interaction. As a final comment, one notes that the coupling constant given by Eq. (16) vanishes in the chiral limit and hence the effective approach is not equivalent to the linear $\sigma$-model, in which this does not happen.

The nonrelativistic potential in configuration space is

$$
\begin{aligned}
V^{S}(x) & =-2 \alpha_{00}^{+} \frac{\mu}{4 \pi}\left[\frac{4 \pi}{\mu^{4}} \int \frac{d^{3} \Delta}{(2 \pi)^{3}} e^{-i \Delta \cdot \mathbf{r}} \sigma\left(-\Delta^{2}\right)\right] \\
& =-\left[3 \alpha_{00}^{+} \frac{\mu}{m}\left(\frac{g}{4 \pi}\right)^{2}\right] \frac{\mu}{4 \pi}\left[S_{c, c}(x)-S_{c, s N}^{(1)}(x)\right],
\end{aligned}
$$

where $x=\mu r$ and

$$
\begin{gathered}
S_{c, c}(x)=\int_{0}^{1} d \alpha \int_{0}^{1} d \beta \frac{\lambda^{2}}{\beta} \frac{e^{-\lambda x}}{x}, \\
S_{c, s N}^{(1)}(x)=\frac{2 m^{2}}{\mu^{2}} \int_{0}^{1} d \alpha \frac{1-\alpha}{\alpha} \int_{0}^{1} d \beta \frac{1-\beta}{\beta} \frac{e^{-\eta x}}{x} .
\end{gathered}
$$




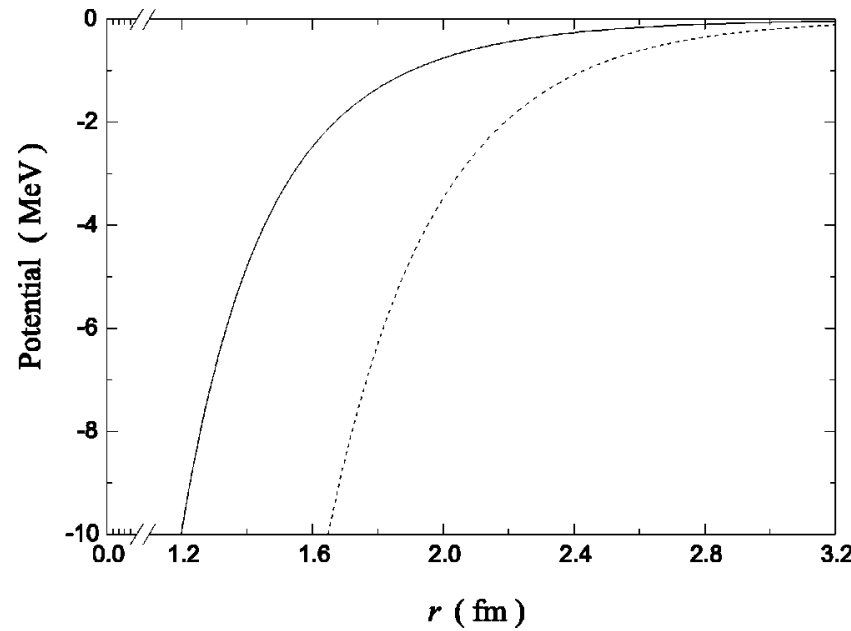

FIG. 3. Scalar-isoscalar potential: asymptotic two-pion exchange (solid line), Eq. (18), and effective scalar exchange (dashed line), Eq. (16), with $\alpha_{00}^{+}=4.61, g_{s}=7.71$, and $m_{s}=483 \mathrm{MeV}$ taken from the last line of Table I.

It is important to note that these functions $S$ are not of the Yukawa type and hence cannot be represented over their full range by terms proportional to $e^{-m_{s} r} / r$, irrespectively of the value chosen for the parameter $m_{s}$. In Fig. 3 we display this potential together with that due to the exchange of an effective scalar meson.

\section{KERNEL}

In this section we construct a kernel for pion production in $N N$ scattering and due to the exchange of two pions. It is represented in Fig. 4, denoted by $\mathcal{T}$, and based on $T_{c b a}$ and $T_{b a}$, the amplitudes for the processes $\pi N \rightarrow \pi \pi N$ and $\pi N$ $\rightarrow \pi N$, respectively. The kernel $\mathcal{T}$ for an outgoing pion with momentum $q$ and isospin index $c$ is
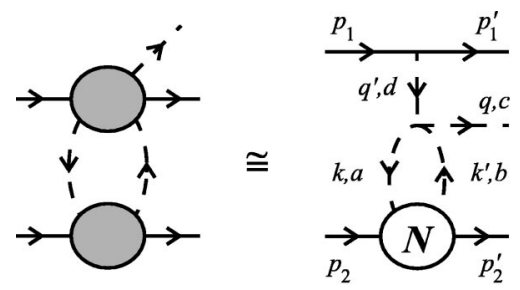

(a)

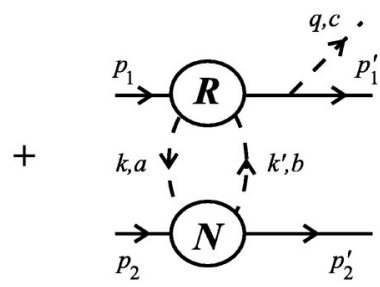

(c)

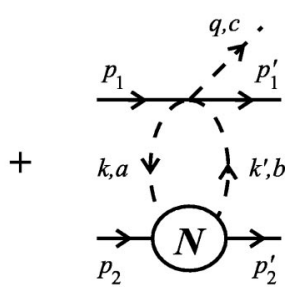

(b)

$$
\mathcal{T}_{c}=-i \frac{1}{2 !} \int \frac{d^{4} Q}{(2 \pi)^{4}} \frac{T_{c b a} T_{b a}}{\left(k^{2}-\mu^{2}\right)\left(k^{\prime 2}-\mu^{2}\right)}
$$

The basic subamplitudes have the isospin structures

$$
\begin{gathered}
T_{b a}=\delta_{a b} T^{+}+i \epsilon_{b a c} \tau_{c} T^{-} \\
T_{c b a}=-i\left\{\delta_{b c} \tau_{a} T_{A}+\delta_{a c} \tau_{b} T_{B}+\delta_{a b} \tau_{c} T_{C}+i \epsilon_{c b a} T_{E}\right\}
\end{gathered}
$$

and hence

$$
\mathcal{T}_{c}=\tau_{c}^{(1)} \mathcal{T}_{1}+i\left(\boldsymbol{\tau}^{(1)} \times \boldsymbol{\tau}^{(2)}\right)_{c} \mathcal{T}_{12}+\tau_{c}^{(2)} \mathcal{T}_{2}
$$

where

$$
\begin{gathered}
\mathcal{T}_{1}=-\frac{1}{2} \int \frac{d^{4} Q}{(2 \pi)^{4}} \frac{\left[T_{A}+T_{B}+3 T_{C}\right]^{(1)}\left[T^{+}\right]^{(2)}}{\left(k^{2}-\mu^{2}\right)\left(k^{\prime 2}-\mu^{2}\right)}, \\
\mathcal{T}_{12}=-\frac{1}{2} \int \frac{d^{4} Q}{(2 \pi)^{4}} \frac{\left[T_{A}-T_{B}\right]^{(1)}\left[T^{-}\right]^{(2)}}{\left(k^{2}-\mu^{2}\right)\left(k^{\prime 2}-\mu^{2}\right)}, \\
\mathcal{T}_{2}=-\frac{1}{2} \int \frac{d^{4} Q}{(2 \pi)^{4}} \frac{2\left[T_{E}\right]^{(1)}\left[T^{-}\right]^{(2)}}{\left(k^{2}-\mu^{2}\right)\left(k^{\prime 2}-\mu^{2}\right)} .
\end{gathered}
$$

We begin by discussing the process $\pi^{b}\left(k^{\prime}\right) N(p)$ $\rightarrow \pi^{a}(k) \pi^{c}(q) N\left(p^{\prime}\right)$. The amplitude $T_{c b a}$ is given by the sum of $T_{c b a}^{\pi}$, a $t$-channel pion-pole contribution, and a remainder, denoted by $\bar{T}_{c b a}$. The explicit forms of these terms, for a system containing just pions and nucleons, was presented in Ref. [36] and here we just quote the main results.

The pion-pole amplitude for on-shell nucleons is

$$
i T_{c b a}^{\pi}=-\frac{m g_{\mathrm{A}}}{f_{\pi}}\left[\bar{u} \tau_{d} \gamma_{5} u\right] \frac{T_{d c b a}^{\pi \pi}}{\left(p^{\prime}-p\right)^{2}-\mu^{2}},
$$

where $f_{\pi}$ and $g_{\mathrm{A}}$ are the pion and axial decay constants, whereas $T_{d c b a}^{\pi \pi}$ is the pion scattering amplitude. At tree level, it is given by

$$
\begin{aligned}
T_{d c b a}^{\pi \pi}= & \frac{1}{f_{\pi}^{2}}\left\{\delta_{a d} \delta_{b c}\left[\left(q-k^{\prime}\right)^{2}-\mu^{2}\right]+\delta_{b d} \delta_{a c}\left[(q+k)^{2}-\mu^{2}\right]\right. \\
& \left.+\delta_{c d} \delta_{a b}\left[\left(k^{\prime}-k\right)^{2}-\mu^{2}\right]\right\}
\end{aligned}
$$

and one has

$$
T_{A}^{\pi}=-\frac{m g_{\mathrm{A}}}{f_{\pi}^{3}} \frac{\left(p^{\prime}-p+k\right)^{2}-\mu^{2}}{\left(p^{\prime}-p\right)^{2}-\mu^{2}} .
$$

The evaluation of $\bar{T}_{c b a}$ requires the calculation of a large number of diagrams. However, long ago Olsson and Turner [37] showed that its leading contribution comes from the effective Lagrangian
FIG. 4. Contributions to the $N N \rightarrow \pi N N$ kernel: (a) pion pole, (b) contact, and (c) and (d) $z$ graphs. 


$$
\overline{\mathcal{L}}=\frac{g_{\mathrm{A}}}{8 f_{\pi}^{3}} \bar{\psi} \gamma_{\mu} \gamma_{5} \boldsymbol{\tau} \psi \cdot \boldsymbol{\phi} \partial^{\mu} \boldsymbol{\phi}^{2}
$$

which gives the following contribution to $\bar{T}_{A}$ :

$$
\bar{T}_{A}=\frac{2 g_{\mathrm{A}}}{8 f_{\pi}^{3}}(2 m+k)
$$

The corresponding expressions for $T_{B}$ and $T_{C}$ are obtained by making $k \rightarrow-k^{\prime}$ and $k \rightarrow q$, respectively.

The main implication of this structure of the $\pi N$ $\rightarrow \pi \pi N$ interaction for our study is that the leading contribution to $\mathcal{T}$ comes from diagrams 4(a) and 4(b). As the $N N$ interaction due to the exchange of two pions is dominated by the scalar-isoscalar channel, in this work we consider only the amplitude $\mathcal{T}_{1}$, Eq. (25), and postpone the discussion of the remaining components to another occasion.

Diagram 4(a) yields

$$
\begin{aligned}
\mathcal{T}_{1}^{\pi}= & {\left[\frac{m g_{\mathrm{A}}}{f_{\pi}^{3}}\right] \frac{1}{\left(p_{1}^{\prime}-p_{1}\right)^{2}-\mu^{2}}\left[\bar{u} \gamma_{5} u\right]^{(1)} \frac{1}{2} \int \frac{d^{4} Q}{(2 \pi)^{4}} } \\
& \times \frac{\left[2\left(p_{1}^{\prime}-p_{1}\right)^{2}+2 q \cdot \Delta+3 \Delta^{2} / 2-5 \mu^{2}+2 Q^{2}\right]\left[T^{+}\right]^{(2)}}{\left[(Q-\Delta / 2)^{2}-\mu^{2}\right]\left[(Q+\Delta / 2)^{2}-\mu^{2}\right]} .
\end{aligned}
$$

We note that the last two terms in the result $Q^{2}=\left(\mu^{2}\right.$ $\left.-\Delta^{2} / 4\right)+\left[(Q-\Delta / 2)^{2}-\mu^{2}\right] / 2+\left[(Q+\Delta / 2)^{2}-\mu^{2}\right] / 2$ allow the cancellation of pion propagators and therefore correspond to short range effects that will be neglected here. We then obtain

$$
\mathcal{T}_{1}^{\pi}=i\left[\frac{m g_{\mathrm{A}}}{f_{\pi}^{3}}\right]\left\{3+\frac{3 q^{2}+4 q \cdot\left(p_{1}^{\prime}-p_{1}\right)}{\left(p_{1}^{\prime}-p_{1}\right)^{2}-\mu^{2}}\right\}\left[\bar{u} \gamma_{5} u\right]^{(1)}\left[\Gamma_{N}^{+}\right]^{(2)},
$$

where $\left[\Gamma_{N}^{+}\right]^{(2)}$ is given by Eq. (5). This result may be associated with the scattering of a pion emitted in one of the nucleons by the pion cloud of the other, indicating that the kernel one is considering here is not fully disentangled from that usually called pion rescattering. Indeed, the description of the rescattering process is based on an intermediate $\pi N$ amplitude for off-shell pions, which satisfies a WardTakahashi identity [39]. In the isospin symmetric channel, this identity may be expressed as

$$
T^{+}\left(q^{\prime 2}, q^{2}\right)=T_{N}^{+}+\frac{q^{\prime 2}+q^{2}-\mu^{2}}{f_{\pi}^{2} \mu^{2}} \sigma(t)[\bar{u} u]+r^{+},
$$

where $T_{N}^{+}$is the nucleon pole (Born) term evaluated with pseudovector coupling, $q$ and $q^{\prime}$ are the momenta of the pions, $\sigma(t)$ is the scalar form factor, and $r^{+}$is a remainder that does not include leading order contributions.

The only term that depends strongly on off-shell effects is that proportional to the scalar form factor and hence one writes

$$
T^{+}\left(q^{\prime 2}, q^{2}\right)=T^{+}\left(\mu^{2}, \mu^{2}\right)+\delta T^{+},
$$

with

$$
\delta T^{+}=\frac{\left(q^{\prime 2}-\mu^{2}\right)+\left(q^{2}-\mu^{2}\right)}{f_{\pi}^{2} \mu^{2}} \sigma(t)[\bar{u} u] .
$$

The contribution of this factor to the pion rescattering amplitude on nucleon 2 reads

$$
\mathcal{T}_{1}^{\delta}=i\left[\frac{m g_{\mathrm{A}}}{f_{\pi}^{3}}\right]\left\{3+\frac{3\left(q^{2}-\mu^{2}\right)}{\left(p_{1}^{\prime}-p_{1}\right)^{2}-\mu^{2}}\right\}\left[\bar{u} \gamma_{5} u\right]^{(1)}\left[\Gamma_{N}^{+}\right]^{(2)}
$$

using Eq. (11). Adding this result to the on-shell $\pi N$ amplitude derived by Gasser, Sainio, and Svarc [Ref. [31], Eq. (A.35)], one recovers Eq. (34). Therefore, in the sequence, we no longer consider the term proportional to the pion pole in that expression, with the understanding that it should be included in the on-shell rescattering amplitude.

The evaluation of diagram 4(b) is more straightforward and produces

$$
\overline{\mathcal{T}}_{1}=-i\left[\frac{m g_{\mathrm{A}}}{f_{\pi}^{3}}\right]\left[\bar{u}\left\{2+\frac{q}{2 m}\right\} \gamma_{5} u\right]^{(1)}\left[\Gamma_{N}^{+}\right]^{(2)}
$$

Using the Goldberger-Treiman relation and Eq. (11), we have

$$
\mathcal{T}_{1}^{\pi}+\overline{\mathcal{T}}_{1}=i\left[\frac{g}{3 \mu^{2} f_{\pi}^{2}}\right] \sigma(t)\left[\bar{u}\left\{1-\frac{q}{2 m}\right\} \gamma_{5} u\right]^{(1)}[\bar{u} u]^{(2)}
$$

One notes that when $\mathcal{T}_{1}^{\pi}$ and $\overline{\mathcal{T}}_{1}$ are added together, a cancellation occurs, which springs from the same mechanism and is very similar to that noticed long ago in the study of exchange currents in pion-deuteron scattering [38].

Another contribution with the same two-pion range comes from diagrams 4(c) and 4(d), which yield

$$
\begin{aligned}
\mathcal{T}_{1}^{z}= & i\left[\frac{g \alpha_{00}^{+}}{m \mu^{3}}\right] \sigma(t)\left[\overline { u } \left\{1-\frac{m \phi}{\left(p^{\prime}+q\right)^{2}-m^{2}}\right.\right. \\
& \left.\left.-\frac{m \phi}{(p-q)^{2}-m^{2}}\right\} \gamma_{5} u\right]^{(1)}[\bar{u} u]^{(2)} .
\end{aligned}
$$

This result includes the propagation of positive energy states that do not contribute to the kernel. Eliminating them and neglecting small noncovariant terms, we have

$$
\mathcal{T}_{1}^{z}=i\left[\frac{g \alpha_{00}^{+}}{m \mu^{3}}\right] \sigma(t)\left[\bar{u}\left\{1+\frac{q}{2 m}\right\} \gamma_{5} u\right]^{(1)}[\bar{u} u]^{(2)} .
$$

Our final expression for the covariant kernel is obtained by adding Eqs. (40) and (42) and reads 


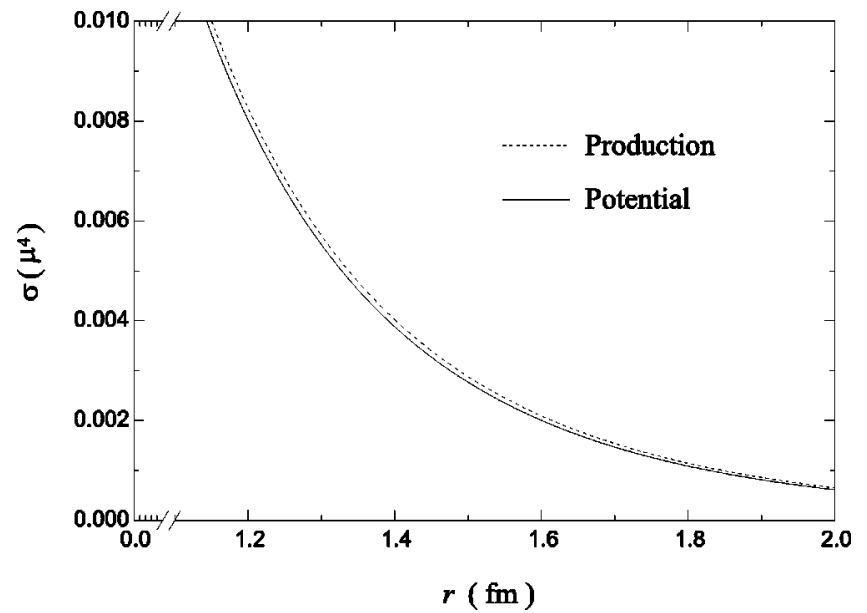

FIG. 5. Fourier transform of $\sigma(t)$, which determines the space dependence of the three-body force and production kernels, as a function of distance $r$.

$$
\begin{aligned}
\mathcal{T}_{1}= & i \sigma(t) g\left\{\left[\frac{1}{3 \mu^{2} f_{\pi}^{2}}+\frac{\alpha_{00}^{+}}{m \mu^{3}}\right] \bar{u} \gamma_{5} u\right. \\
& \left.-\left[\frac{1}{3 \mu^{2} f_{\pi}^{2}}-\frac{\alpha_{00}^{+}}{m \mu^{3}}\right] \bar{u} \frac{q}{2 m} \gamma_{5} u\right\}^{(1)}[\bar{u} u]^{(2)} .
\end{aligned}
$$

This covariant amplitude is our main result.

\section{APPLICATION}

In order to consider applications in low energy processes, we perform a nonrelativistic approximation in our results. In the case of the central potential, Eq. (11), one has

$$
t^{S}=2 \alpha_{00}^{+} \sigma(t) / \mu^{3},
$$

where $t=-\Delta^{2}$ and we have discarded a normalization factor $4 \mathrm{~m}^{2}$. On the other hand, the kernel, suited to be used with nuclear wave functions, is

$$
\begin{aligned}
t_{1}= & i \sigma(t) \frac{g}{2 m}\left\{-\left[\frac{1}{3 \mu^{2} f_{\pi}^{2}}+\frac{\alpha_{00}^{+}}{m \mu^{3}}\right] \boldsymbol{\sigma} \cdot\left(\boldsymbol{p}^{\prime}-\boldsymbol{p}\right)\right. \\
& \left.+\left[\frac{1}{3 \mu^{2} f_{\pi}^{2}}-\frac{\alpha_{00}^{+}}{m \mu^{3}}\right] \boldsymbol{\sigma} \cdot \boldsymbol{q}\right\}^{(1)} .
\end{aligned}
$$

The term proportional to $\boldsymbol{\sigma} \cdot\left(\boldsymbol{p}^{\prime}-\boldsymbol{p}\right) / 3 \mu^{2} f_{\pi}^{2}$ in this result coincides with that produced recently in Ref. [27].

As discussed in Refs. [21] and [22], a pion production kernel such as $t_{1}$ gives rise to three-body forces and again one has $t \cong-\Delta^{2}$. In the case of threshold pion production, on the other hand, $t \cong \mu^{2} / 4-\Delta^{2}$. In order to test the influence of these different values of $t$, in Fig. 5 we plot the Fourier transform of the function $\sigma(t)$, which dictates the space dependence of the kernel in the two cases. Inspecting it, one learns that the energy component of the four momentum transferred has little importance and hence the static result also holds for the production kernel. This allows one to relate it directly to the central potential

$$
\begin{aligned}
t_{1}= & i \frac{g_{\mathrm{A}}}{f_{\pi}} \frac{t^{S}}{2 m}\left\{-\left[\frac{\mu m}{6 \alpha_{00}^{+} f_{\pi}^{2}}+\frac{1}{2}\right] \boldsymbol{\sigma} \cdot\left(\boldsymbol{p}^{\prime}-\boldsymbol{p}\right)\right. \\
& \left.+\left[\frac{\mu m}{6 \alpha_{00}^{+} f_{\pi}^{2}}-\frac{1}{2}\right] \boldsymbol{\sigma} \cdot \boldsymbol{q}\right\}
\end{aligned}
$$

In order to use these results in actual calculations, in either momentum or configuration space, one has to evaluate the function $\sigma(t)$ numerically and, then, the sandwich of the kernel between two-nucleon wave functions. Since the kernel and the central potential are closely related, consistency would require that the same dynamics should be used in the construction of both the operator $t_{1}$ and the wave functions. However, at present, the potential due to the exchange of two pions is reliable at large distances only and hence it is not suited to determine wave functions by means of the Schrödinger equation. Therefore, the possibility of using Eq. (46) with one's favorite scalar potential is an interesting one.

\section{SUMMARY AND CONCLUSIONS}

In this work we have shown that the central component of the $N N$ potential at large distances, which is due to the exchange of two uncorrelated pions, may be naturally expressed in terms of $\sigma(t)$, the scalar form factor. This function is related to the $\pi N \sigma$-term and may, if one wishes, be parametrized as an effective scalar meson exchange. However, the coupling of this state to nucleons vanishes in the chiral limit and hence this scalar meson does not correspond to that present in the linear $\sigma$-model.

We have also obtained a two-pion exchange kernel for the process $N N \rightarrow \pi N N$, which can be applied in both threebody forces and pion production in $N N$ scattering. The complete calculation of this kernel would require the evaluation of a large number of diagrams. Thus, in order to estimate the dominant term at large $N N$ distances, we have used just the leading contributions to the subamplitudes $\pi N \rightarrow \pi N$ and $\pi N \rightarrow \pi \pi N$, in the framework of chiral symmetry. The simplified result so obtained involves a cancellation between contact-three-pion and pion-pole vertices. The latter may also be associated with an off-shell intermediate $\pi N$ amplitude and has been included into the Tucson-Melbourne [40] two-pion exchange three-nucleon potential. This means that this force does include a term describing a two-pion exchange between a pair of nucleons. Thus, the use of an onshell $\pi N$ amplitude gives rise to a less ambiguous definition of the two-pion exchange three-body force [41].

At large distances, the kernel is closely related to the twopion exchange scalar isoscalar $N N$ potential. Indeed, in the case of three-body forces, we could show that the kernel and the potential have the same spatial dependence. For threshold pion production, this relationship is also approximately valid. These results led us to produce expressions that relate directly the kernel to the potential. Using the extreme numerical values for $\alpha_{00}^{+}$found in the literature, namely, 3.68 [29] 
and 6.74 [33], in Eq. (46), one has

$$
t_{1}=i \frac{g_{\mathrm{A}}}{f_{\pi}} \frac{t^{S}}{2 m}\left\{-1.18 \boldsymbol{\sigma} \cdot\left(\boldsymbol{p}^{\prime}-\boldsymbol{p}\right)+0.18 \boldsymbol{\sigma} \cdot \boldsymbol{q}\right\}^{(1)}
$$

or

$$
t_{1}=i \frac{g_{\mathrm{A}}}{f_{\pi}} \frac{t^{S}}{2 m}\left\{-0.87 \boldsymbol{\sigma} \cdot\left(\boldsymbol{p}^{\prime}-\boldsymbol{p}\right)-0.13 \boldsymbol{\sigma} \cdot \boldsymbol{q}\right\}^{(1)} .
$$

These results are quite close to the kernel obtained by ourselves sometime ago [22], given by

$$
t_{1}=i \frac{g_{\mathrm{A}}}{f_{\pi}} \frac{t^{S}}{2 m}\left\{-\boldsymbol{\sigma} \cdot\left(\boldsymbol{p}^{\prime}-\boldsymbol{p}\right)\right\}^{(1)},
$$

in the case of a model based on effective scalar-isoscalar mesons. ${ }^{1}$ This allows one to consider the relationship between the kernel and the potential to be a rather general one. The reason for this generality springs from an old insight by Nambu and co-workers [42] and Weinberg [43] that, for generic states $A$ and $B$, the leading contributions to the process $A \rightarrow \pi B$ are obtained by inserting the pion, with gradient coupling, into the external lines of the process $A \rightarrow B$.

\footnotetext{
${ }^{1}$ In that work we used $g_{A}=1$.
}

Finally, we would like to point out that we may expect the contributions from the kernel $t_{1}$ to be large. In order to see this, note that momentum conservation allows one to write

$$
t_{1}=i \frac{g_{\mathrm{A}}}{f_{\pi}} \frac{t^{S}}{2 m}\{\boldsymbol{\sigma} \cdot(\boldsymbol{q}-\boldsymbol{\Delta})\}^{(1)}
$$

and, in the case of threshold pion production, in configuration space one has

$$
t_{1}=\frac{g_{\mathrm{A}}}{f_{\pi}} \frac{\mu}{2 m} \boldsymbol{\sigma}^{(1)} \cdot \nabla_{x} V^{S}(x) .
$$

As the central potential contains Yukawa functions with effective masses which are not small, its gradient produces a large kernel, proportional to those masses.

\section{ACKNOWLEDGMENTS}

We thank Bira van Kolck and Carlos A. da Rocha for useful conversations. C.M.M. thanks the hospitality of the Instituto de Física Teórica (Universidade Estadual Paulista) in the initial stage of this work and acknowledges the support of FAPESP (Brazilian Agency, Grant No. 99/00080-5) and NSF (Grant No. PHY_94-20470). J.C.P. acknowledges the support of FAPESP (Brazilian Agency, Grant No. 94/034697).
[1] D. A. Hutcheo et al., Nucl. Phys. A535, 618 (1991).

[2] H. O. Meyer et al., Phys. Rev. Lett. 65, 2846 (1990); Nucl. Phys. A539, 633 (1992).

[3] A. Bondar et al., Phys. Lett. B 356, 8 (1995).

[4] M. Drochner et al., Phys. Rev. Lett. 77, 454 (1996).

[5] P. Heimberg et al., Phys. Rev. Lett. 77, 1012 (1996).

[6] W. W. Daehnick et al., Phys. Rev. Lett. 74, 2913 (1995); Phys. Lett. B 423, 213 (1998); J. G. Hardie et al., Phys. Rev. C 56, 20 (1997); R. W. Flammang et al., ibid. 58, 916 (1998).

[7] D. S. Koltun and A. Reitan, Phys. Rev. 141, 1413 (1966); Nucl. Phys. B4, 629 (1968).

[8] G. A. Miller and P. U. Sauer, Phys. Rev. C 44, 1725 (1991).

[9] F. Hachenberg and H. J. Pirner, Ann. Phys. (N.Y.) 112, 401 (1978).

[10] V. P. Efrosinin, D. A. Zaikin, and I. I. Osipchuk, Z. Phys. A 322, 573 (1985); Phys. Lett. B 246, 10 (1990).

[11] E. Hernández and E. Oset, Phys. Lett. B 350, 158 (1995).

[12] C. Hanhart, J. Haidenbauer, A. Reuber, C. Schütz, and J. Speth, Phys. Lett. B 358, 21 (1995); J. Haidenbauer, C. Hanhart, and J. Speth, Acta Phys. Pol. B 27, 2893 (1996).

[13] T. D. Cohen, J. L. Friar, G. A. Miller, and U. van Kolck, Phys. Rev. C 53, 2661 (1996).

[14] U. van Kolck, G. A. Miller, and D. O. Riska, Phys. Lett. B 388, 679 (1996).

[15] C. A. da Rocha, G. A. Miller, and U. van Kolck, Phys. Rev. C 61, 034613 (2000).

[16] C. Hanhart, J. Haidenbauer, M. Hoffmann, U. -G. Meißner, and J. Speth, Phys. Lett. B 424, 8 (1998).
[17] T.-S. H. Lee and D. O. Riska, Phys. Rev. Lett. 70, 2237 (1993).

[18] J. Adam, A. Stadler, M. T. Peña, and F. Gross, Phys. Lett. B 407, 97 (1997).

[19] C. Hanhart, J. Haidenbauer, O. Krehl, and J. Speth, Phys. Lett. B 444, 25 (1998).

[20] M. T. Peña, D. O. Riska, and A. Stadler, Phys. Rev. C 60, 045201 (1999).

[21] S. A. Coon, M. T. Peña, and D. O. Riska, Phys. Rev. C 52, 2925 (1995).

[22] C. M. Maekawa and M. R. Robilotta, Phys. Rev. C 57, 2839 (1998).

[23] C. Ordóñez, L. Ray, and U. van Kolck, Phys. Rev. Lett. 72, 1982 (1994); Phys. Rev. C 53, 2086 (1996).

[24] M. R. Robilotta and C. A. da Rocha, Nucl. Phys. A615, 391 (1997); M. R. Robilotta, ibid. A595, 171 (1995).

[25] N. Kaiser, R. Brockman, and W. Weise, Nucl. Phys. A625, 758 (1997); N. Kaiser, S. Gertendörfer, and W. Weise, ibid. A637, 395 (1998).

[26] C. M. Maekawa and C. A. da Rocha, nucl-th/9905052.

[27] V. Bernard, N. Kaiser, and U.-G. Meißner, Eur. Phys. J. A 4, 259 (1999).

[28] V. Dmitrašinović, K. Kubodera, F. Myhrer, and T. Sato, Phys. Lett. B 465, 43 (1999).

[29] G. Höhler, in Numerical data and Functional Relationships in Science and Technology, edited by H. Schopper, LandöltBernstein, New Series, Group I, Vol. 9b, Pt. 2 (SpringerVerlag, Berlin, 1983). 
[30] M. R. Robilotta (unpublished).

[31] J. Gasser, M. E. Sainio, and A. Švarc, Nucl. Phys. B307, 779 (1988)

[32] J. Gasser, H. Leutwyler, and M. E. Sainio, Phys. Lett. B 253, 252 (1991); 253, 260 (1991).

[33] W. B. Kaufmann and G. E. Hite, Phys. Rev. C 60, 055204 (1999).

[34] M. M. Pavan, R. A. Arndt, I. I. Strakovsky, and R. L. Workman, $\pi N$ Newsletter 15, 118 (1999).

[35] R. Machleidt, K. Holinde, and C. Elster, Phys. Rep. 149, 1 (1987).

[36] J. C. Pupin and M. R. Robilotta, Phys. Rev. C 60, 014003 (1999).

[37] M. G. Olsson and L. Turner, Phys. Rev. Lett. 20, 1127 (1968).
[38] M. R. Robilotta and C. Wilkin, J. Phys. G 4, L115 (1978).

[39] L. S. Brown, W. J. Pardee, and R. D. Peccei, Phys. Rev. D 4, 2801 (1971).

[40] S. A. Coon, M. S. Scadron, and B. R. Barrett, Nucl. Phys. A242, 467 (1975); S. A. Coon, M. S. Scadron, P. C. McNamee, B. R. Barrett, D. W. E. Blatt, and B. H. J. McKellar, ibid. A317, 242 (1979); S. A. Coon and W. Glöckle, Phys. Rev. C 23, 1790 (1981).

[41] H. T. Coelho, T. K. Das, and M. R. Robilotta, Phys. Rev. C 28, 1812 (1983); M. R. Robilotta, and H. T. Coelho, Nucl. Phys. A460, 645 (1986).

[42] Y. Nambu and D. Lurie, Phys. Rev. 125, 1429 (1962); Y. Nambu and E. Schrauner, ibid. 128, 862 (1962).

[43] S. Weinberg, Phys. Rev. Lett. 16, 879 (1966). 